\title{
On the Abaqus FEA model of finite viscoelasticity
}

\author{
Jacopo Ciambella ${ }^{a}$, Michel Destrade ${ }^{b}$, Ray W. Ogden ${ }^{c}$ \\ ${ }^{a}$ Department of Structural and Geotechnical Engineering, \\ Università di Roma "La Sapienza", \\ Via Eudossiana, 18 - 00184 - Roma, Italy. \\ ${ }^{b}$ Institut Jean Le Rond d'Alembert, \\ CNRS/Université Pierre et Marie Curie, \\ Case 162, 75252 Paris Cedex 05, France. \\ ${ }^{c}$ Department of Mathematics, \\ University of Glasgow, \\ Glasgow G128QW, United Kingdom.
}

\begin{abstract}
Predictions of the QLV (Quasi-Linear Viscoelastic) constitutive law are compared with those of the ABAQUS viscoelastic model for two simple motions in order to highlight, in particular, their very different dissipation rates and certain shortcomings of the ABAQUS model.
\end{abstract}




\section{Introduction}

As the demand for fuel efficient cars intensifies, tyre manufacturers try harder to estimate accurately the energy losses of their products. However the mechanics of tires are a most complex topic due to the variety of mechanical factors involved, such as nonlinear responses, structural inhomogeneities, complicated geometries and solicitations, hypo-, visco-, and hyperelasticity, etc. Consequently, advanced finite element codes are often called to the rescue in order to simulate real-world situations, and to evaluate the energy efficiency of a tyre. These commercial codes are often used as a "black box", and the validity of the answer is rarely put into question, even though it might provide a decisive argument in favor of, or against, the viability of a given tyre model.

Here we examine the current implementation of nonlinear viscoelastic effects in the Abaqus Finite Element Analysis (FEA) package. We find that it is not sound physically, and that it gives results that are not consistent with the thermomechanically-based Quasi Linear Viscoeleastic (QLV) model. We present both models in the next section, and highlight their main differences. Then we investigate two toy experiments, using the incompressible neo-Hookean material for the elastic response. We consider the equi-biaxial extension test in Section 3 , and the simple shear test in Section 4. In both cases, the Abaqus FEA model predicts higher energy losses than the QLV model.

\section{Abaqus FEA finite viscoelasticity model}

First we introduce some notations. We call $\boldsymbol{F}$ the deformation gradient; it is defined as $\boldsymbol{F}=\partial \boldsymbol{x} / \boldsymbol{X}$, where $\boldsymbol{x}$ is the coordinate in the current configuration of a particle at $\boldsymbol{X}$ in the reference configuration. We call $J$ its determinant: $J=\operatorname{det} \boldsymbol{F}$, and $\boldsymbol{B} \equiv \boldsymbol{F} \boldsymbol{F}^{T}, \boldsymbol{C} \equiv \boldsymbol{F}^{T} \boldsymbol{F}$ the associated Cauchy-Green strain tensors, where a superscript $T$ denotes the transpose. We also introduce the velocity gradient $\boldsymbol{D} \equiv$ $\left[\dot{\boldsymbol{F}} \boldsymbol{F}^{-1}+\left(\dot{\boldsymbol{F}} \boldsymbol{F}^{-1}\right)^{T}\right] / 2$, where the superimposed dot represents the time derivative. When a solid is incompressible, every deformation is isochoric, so that

$$
J=1, \quad \operatorname{tr} \boldsymbol{D}=0 .
$$

We recall that the dissipated power per unit volume is $\boldsymbol{\sigma}(t) \cdot \boldsymbol{D}(t) \equiv \operatorname{tr}(\boldsymbol{\sigma}(t) \boldsymbol{D}(t))$, so that $E_{d}$, the dissipated energy per unit volume over a period $T$, is given by

$$
E_{d}=\frac{1}{T} \int_{0}^{T} \boldsymbol{\sigma}(t) \cdot \boldsymbol{D}(t) \mathrm{d} t
$$


Next we present the Abaqus FEA model. Section 4.8.2 of the Abaqus Theory Manual (Hibbit et al. 2007) gives the following constitutive relation to model nonlinear viscoelastic effects,

$$
\boldsymbol{\sigma}(t)=\boldsymbol{\sigma}_{e}(t)+\operatorname{SYM}\left\{\boldsymbol{F}(t)\left[\int_{0}^{t} \frac{J(s)}{J(t)} \dot{G}(t-s) \boldsymbol{F}^{-1}(s) \boldsymbol{\sigma}_{e}(s) \boldsymbol{F}(s) \mathrm{d} s\right] \boldsymbol{F}^{-1}(t)\right\},
$$

where $\boldsymbol{\sigma}_{e}$ is the instantaneous Cauchy stress response (elastic response at very short times) and $G$ is the so-called memory kernel, characterizing the stress relaxation (with $G(0)=1$ ). Also, "SYM" denotes the symmetric part of the bracketed term; hence $D=\operatorname{SYM}\left\{\dot{\boldsymbol{F}} \boldsymbol{F}^{-1}\right\}$. This constitutive relation is valid for compressible as well as incompressible solids, because in that latter case the hydrostatic term $-\hat{\boldsymbol{p}} \boldsymbol{I}$ in $\boldsymbol{\sigma}_{e}$ (where $\hat{p}$ is a Lagrange multiplier) does not produce work, neither in the instantaneous response, nor in the history term, as expected.

For incompressible solids, $J=1$ at all times and $\boldsymbol{\sigma}_{e}$ has the general form:

$$
\boldsymbol{\sigma}_{e}=-\hat{p} \boldsymbol{I}+\psi_{1} \boldsymbol{B}+\psi_{2} \boldsymbol{B}^{2}
$$

where $\psi_{1}, \psi_{2}$ are scalar functions of $t$ and of the first and second principal strain invariants. Then (3) reduces to

$$
\begin{aligned}
\boldsymbol{\sigma}(t)=-p(t) \boldsymbol{I}+ & \psi_{1}(t) \boldsymbol{B}(t)+\psi_{2}(t) \boldsymbol{B}(t)^{2} \\
& +\sum_{i=1}^{2} \operatorname{SYM}\left\{\boldsymbol{F}(t)\left[\int_{0}^{t} \dot{\boldsymbol{G}}(t-s) \psi_{i}(s) \boldsymbol{C}(s)^{i} \mathrm{~d} s\right] \boldsymbol{F}^{-1}(t)\right\},
\end{aligned}
$$

where $p(t)=\hat{p}(t)+\int_{0}^{t} \dot{G}(t-s) \hat{p}(s) \mathrm{d} s$ is arbitrary and remains to be determined from initial/boundary conditions.

The Abaqus FEA model is reminiscent of, and similar to a well-established model of finite viscoelasticity, namely the Pipkin-Rogers model (Pipkin and Rogers 1968), which reads in the incompressible case as (Wineman 1972, Johnson et al. 1996)

$$
\boldsymbol{\sigma}(t)=-p(t) \boldsymbol{I}+\boldsymbol{F}(t)\left\{\boldsymbol{R}[\boldsymbol{C}(t), 0]+\int_{0}^{t} \frac{\partial}{\partial(t-s)}(\boldsymbol{R}[\boldsymbol{C}(s), t-s]) \mathrm{d} s\right\} \boldsymbol{F}(t)^{T} .
$$

Here $p$ is a Lagrange multiplier resulting from the internal constraint of incompressibility and $R$ is a strain dependent tensorial relaxation function, with the general form

$$
\boldsymbol{R}=\phi_{0} \boldsymbol{I}+\phi_{1} \boldsymbol{C}+\phi_{2} \boldsymbol{C}^{2}
$$


where $\phi_{0}, \phi_{1}, \phi_{2}$ are scalar functions of $t$ and of the first and second principal strain invariants.

By an appropriate choice of the $\phi_{i}$, the Pipkin-Rogers model reduces to the so-called Quasi-Linear Viscoelastic (QLV) model, which has proved to be a most successful phenomenological model for the behavior of non-linear viscoelastic solids, see references in (Johnson et al. 1996, Wineman and Rajagopal 2008). Equation (6) is derived rigorously by successive approximations from the basic physical requirements governing the behavior of solids with memory (such as the principle of determinism and local action, the principle of material objectivity, etc.), see the review (Drapaca et al. 2007).

However we notice upon inspection of (5) and (6) that there are at least two differences between the models suggesting that the Abaqus FEA model does not rely on physical principles. First, the integral term in equation (3) is generally nonsymmetric, in contrast with the integral term in equation (6). This is taken care of, in a somewhat arbitrary and ad hoc manner, by using the "SYM" operator. Also, the history (time integral) term in the Abaqus FEA model terminates with $\boldsymbol{F}(t)^{-1}$ in contrast with the history term in the the QLV model, which terminates with $\boldsymbol{F}(t)^{T}$.

To emphasize the differences between each model, we henceforth focus on a viscoelastic incompressible solid which has an instantaneous response modeled by the neo-Hookean stress-strain relationship (already implemented into Abaqus),

$$
\boldsymbol{\sigma}_{e}=-p \boldsymbol{I}+\mu_{0} \boldsymbol{B}
$$

where $\mu_{0}>0$ is a constant, the initial shear modulus. Also, the time relaxation of the solid is assumed to be governed by a one-term Prony series expansion, so that after an infinite time the shear modulus settles at the value $\mu_{\infty}$, say:

$$
G(t)=\frac{\mu_{\infty}}{\mu_{0}}+\left(1-\frac{\mu_{\infty}}{\mu_{0}}\right) \mathrm{e}^{-t / \tau}
$$

In that case, the Abaqus FEA model gives the identifications $\psi_{1}=\mu_{0}, \psi_{2}=0$, so that

$\boldsymbol{\sigma}(t)=-p(t) \boldsymbol{I}+\mu_{0} \boldsymbol{B}(t)+\left(\mu_{\infty}-\mu_{0}\right) \operatorname{SYM}\left\{\boldsymbol{F}(t)\left[\int_{0}^{t} \frac{\mathrm{e}^{-(t-s) / \tau}}{\tau} \boldsymbol{C}(s) \mathrm{d} s\right] \boldsymbol{F}^{-1}(t)\right\}$.

In contrast, the QLV model gives the identifications $\phi_{0}=\mu_{0} G, \phi_{1}=0, \phi_{2}=0$, and the history term turns out to be integrable, to give eventually

$$
\boldsymbol{\sigma}(t)=-p(t) \boldsymbol{I}+\left[\mu_{\infty}+\left(\mu_{0}-\mu_{\infty}\right) \mathrm{e}^{-t / \tau}\right] \boldsymbol{B}(t) .
$$


Here the viscoelastic effect is at its most limpid, whereas it seems quite complicated in (10). We now look at two simple examples of motions.

\section{Equi-biaxial extension}

The equi-biaxial extension test of an incompressible solid is described by the following motion,

$$
x_{1}=\lambda(t) X_{1}, \quad x_{2}=\lambda(t)^{-1 / 2} X_{2}, \quad x_{3}=\lambda(t)^{-1 / 2} X_{3},
$$

where $\lambda(t)$ is the stretch ratio in the direction of extension. Then clearly the deformation gradient is diagonal,

$$
\boldsymbol{F}(t)=\operatorname{Diag}\left[\lambda(t), \lambda(t)^{-1 / 2}, \lambda(t)^{-1 / 2}\right] .
$$

Assuming now that the solid is subject to uni-axial tension: $\sigma_{[11]} \neq 0, \sigma_{[22]}=$ $\sigma_{[33]}=0$, allows us to compute the Lagrange multiplier $p$. Then we find the resulting Cauchy stress non-zero component is

$$
\sigma_{[11]}(t)=\mu_{0}\left[\lambda^{2}(t)-\lambda(t)^{-1}\right]+\left(\mu_{\infty}-\mu_{0}\right) \int_{0}^{t} \frac{\mathrm{e}^{-(t-s) / \tau}}{\tau}\left[\lambda^{2}(s)-\lambda(s)^{-1}\right] \mathrm{d} s,
$$

for the Abaqus FEA model, and

$$
\sigma_{[11]}(t)=\left[\mu_{\infty}+\left(\mu_{0}-\mu_{\infty}\right) \mathrm{e}^{-t / \tau}\right]\left[\lambda^{2}(t)-\lambda(t)^{-1}\right],
$$

for the QLV model. The difference between each model is now clearly apparent, and it reflects on the dissipated power, as we know confirm numerically.

From an experimental point of view, it is common practice to have a dynamic displacement superimposed on a large static deformation. Here we consider that the neo-Hookean solid is strained in equi-biaxial tension by $30 \%$ from time $t=0$ to time $t=1$, and then made to oscillate with an amplitude of $20 \%$ :

$$
\lambda(t)=\left\{\begin{array}{l}
1+0.3 t, \quad 0 \leq t \leq 1, \\
1.3+0.2 \sin \omega(t-1), \quad t>1
\end{array}\right.
$$

For the remaining parameters we pick

$$
\mu_{\infty} / \mu_{0}=0.5, \quad \tau=0.1 \mathrm{~s}, \quad \omega=10.0 \mathrm{~s}^{-1},
$$




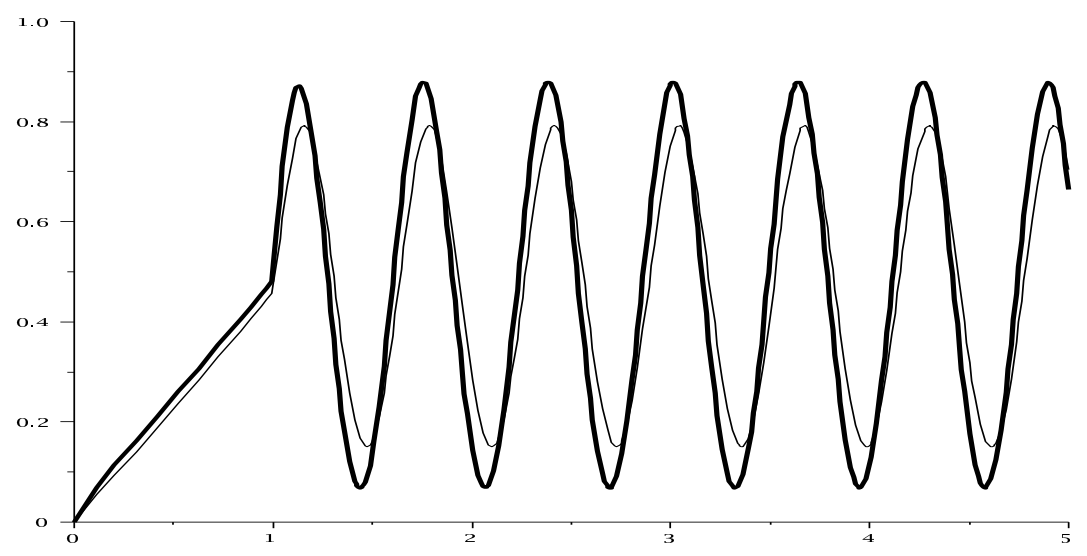

Figure 1: Variations of the axial tension $\sigma_{[11]}$ with time, for the Abaqus FEA model (thick curve) and for the QLV model (thin curve) in the case of equi-biaxial tension.

or we keep $\omega$ as a free parameter when we investigate the frequency dependence of the dissipated energy.

Hence Figure 1 shows that at those values, the predictions from the Abaqus model differ widely from those of the QLV model.

The numerical simulations are performed with Abaqus 6.7-1 on a bidimensional square element of $1 \mathrm{~m}$ side; we use a single CPS4 element (4 nodes, bilinear, plane stress) with an implicit solution scheme. Figure 2 shows clearly that the Abaqus model overestimates the energy dissipation compared to the QLV model.

\section{Simple Shear}

We now consider a simple shear of amount $\gamma$ say,

$$
x_{1}=X_{1}+\gamma(t) X_{2}, \quad x_{2}=X_{2}, \quad x_{3}=X_{3},
$$

for which the deformation gradient is not symmetric,

$$
\boldsymbol{F}(t)=\left[\begin{array}{ccc}
1 & \gamma(t) & 0 \\
0 & 1 & 0 \\
0 & 0 & 1
\end{array}\right]
$$

Simple calculations reveal that a sheared solid described by the Abaqus FEA model or by the QLV model is in a state of plane stress $\left(\sigma_{[i j]} \neq 0\right.$ for $i, j=1,2$; 


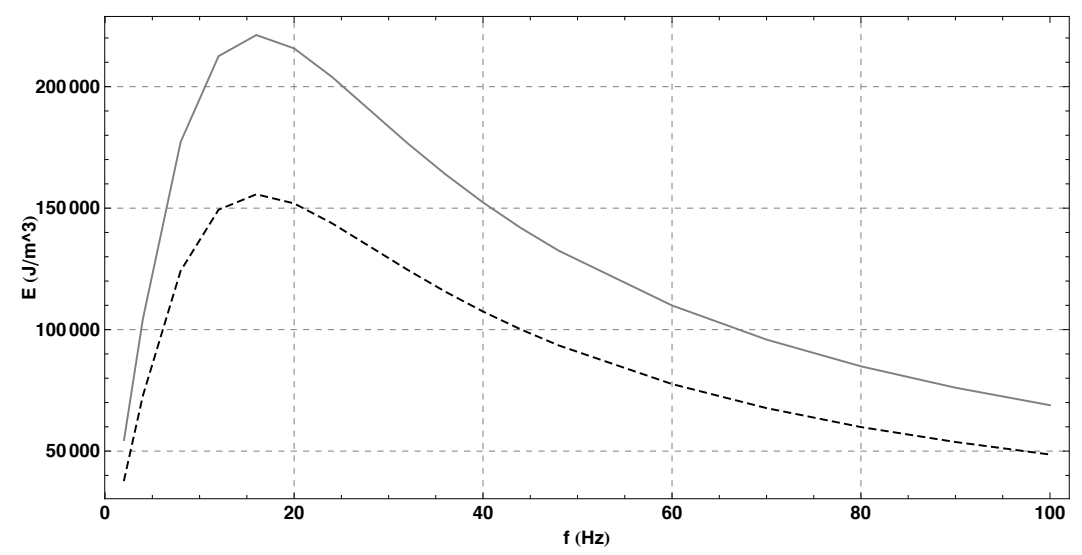

Figure 2: Energy dissipation per unit volume over a single period for the Abaqus model (gray, solid curve) and for the QLV model (black, dashed curve) in the case of equi-biaxial tension.

$\sigma_{[3 j]}=0$ for $\left.j=1,2,3\right)$ when the Lagrange multiplier $p$ is taken as

$$
p(t)=\mu_{\infty}-\left(\mu_{\infty}-\mu_{0}\right) \mathrm{e}^{-t / \tau}
$$

Then we find for the Abaqus FEA model,

$$
\begin{aligned}
& \sigma_{[11]}(t)=\left(\mu_{\infty}-\mu_{0}\right) \int_{0}^{t} \frac{\mathrm{e}^{-(t-s) / \tau}}{\tau} \gamma(s) \gamma(t) \mathrm{d} s \\
& \sigma_{[12]}(t)=\mu_{0} \gamma(t)+\left(\mu_{\infty}-\mu_{0}\right) \int_{0}^{t} \frac{\mathrm{e}^{-(t-s) / \tau}}{2 \tau} \gamma(s)\left[2+\gamma(s) \gamma(t)-\gamma(t)^{2}\right] \mathrm{d} s \\
& \sigma_{[22]}(t)=\left(\mu_{\infty}-\mu_{0}\right) \int_{0}^{t} \frac{\mathrm{e}^{-(t-s) / \tau}}{\tau} \gamma(s)[\gamma(s)-\gamma(t)] \mathrm{d} s
\end{aligned}
$$

and for the QLV model

$$
\begin{aligned}
& \sigma_{[11]}(t)=\left[\mu_{\infty}-\left(\mu_{\infty}-\mu_{0}\right) \mathrm{e}^{-t / \tau}\right] \gamma(t)^{2} \\
& \sigma_{[12]}(t)=\left[\mu_{\infty}-\left(\mu_{\infty}-\mu_{0}\right) \mathrm{e}^{-t / \tau}\right] \gamma(t), \\
& \sigma_{[22]}(t)=0
\end{aligned}
$$

These latter expressions are intuitively expected for the neo-Hookean solid, because for its instantaneous response at very short times, the Cauchy stress has components $\sigma_{e[11]}=\mu_{0} \gamma^{2}, \sigma_{e[12]}=\mu_{0} \gamma, \sigma_{e[22]}=0$. In contrast, the Abaqus FEA 


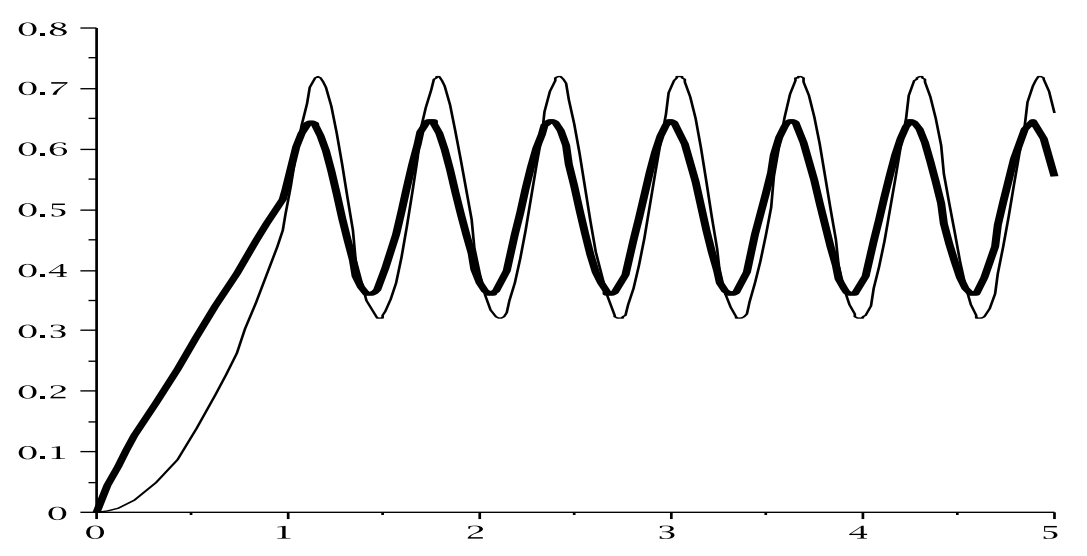

Figure 3: Variations of $\sigma_{[12]}(t) / \mu_{0}$ for the Abaqus analytical model (thick curve) and the QLV model (thin curve).

model gives rise to a $\sigma_{[22]}$ component purely due to viscoelastic effects. This behavior is clearly non-physical, for in the case of a quasi-static loading, no traction $\sigma_{[22]}$ is required to shear a neo-Hookean solid, and yet the relaxation process would create such a component ex nihilo! These discrepancies have grave consequences for the dissipated power because here

$$
\boldsymbol{D}(t)=\left[\begin{array}{ccc}
0 & \dot{\gamma}(t) / 2 & 0 \\
\dot{\gamma}(t) / 2 & 0 & 0 \\
0 & 0 & 0
\end{array}\right], \quad \text { so that } \quad \boldsymbol{\sigma} \cdot \boldsymbol{D}=\dot{\gamma}(t) \sigma_{[12]}(t),
$$

which is obviously not the same depending on the model considered.

We confirm these findings by testing the Abaqus FEA software against the current formulas (21)-(23). We take the amount of shear $\gamma(t)$ to vary as

$$
\gamma(t)=\left\{\begin{array}{l}
t, \quad 0 \leq t \leq 1 \\
1+0.2 \sin [\omega(t-1)], \quad t>1,
\end{array}\right.
$$

with the other parameters given by (17). Figures 3 and 4 display the variations of the $\sigma_{[12]}$ and $\sigma_{[12]}$ components computed from (21) and (22). Finally, we find that the Abaqus model overestimates the energy dissipation with respect to the QLV model, see Figure 5. Using the commercial software simulation, we recovered the thick curves, which confirms our opinion that equation (3) is actually implemented in the commercial code.. 


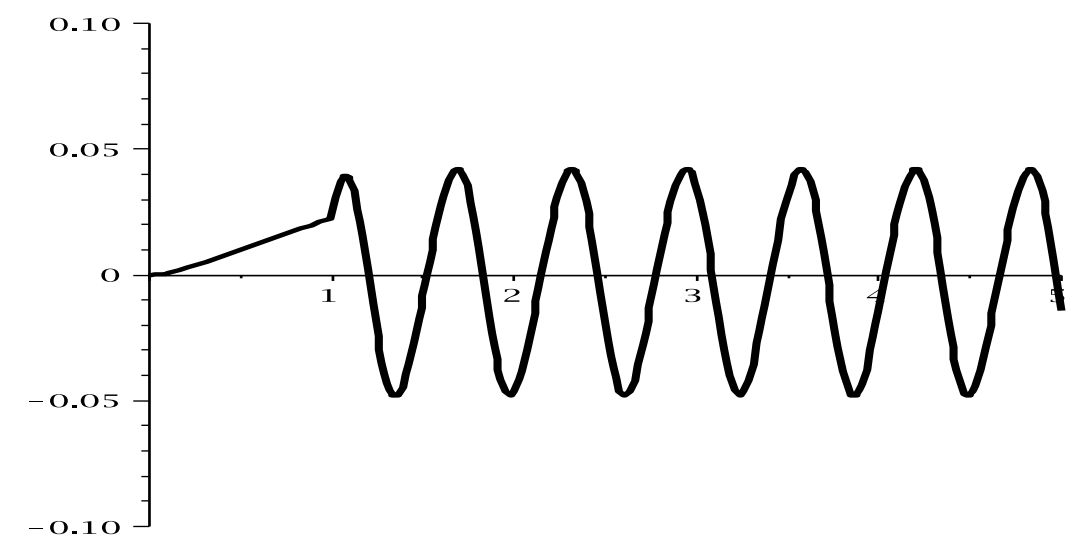

Figure 4: Variations of $\sigma_{[22]}(t) / \mu_{0}$ for the Abaqus FEA model (thick curve) and the QLV model (thin curve). The latter does not show clearly on the graph, because it is equal to zero at all times.

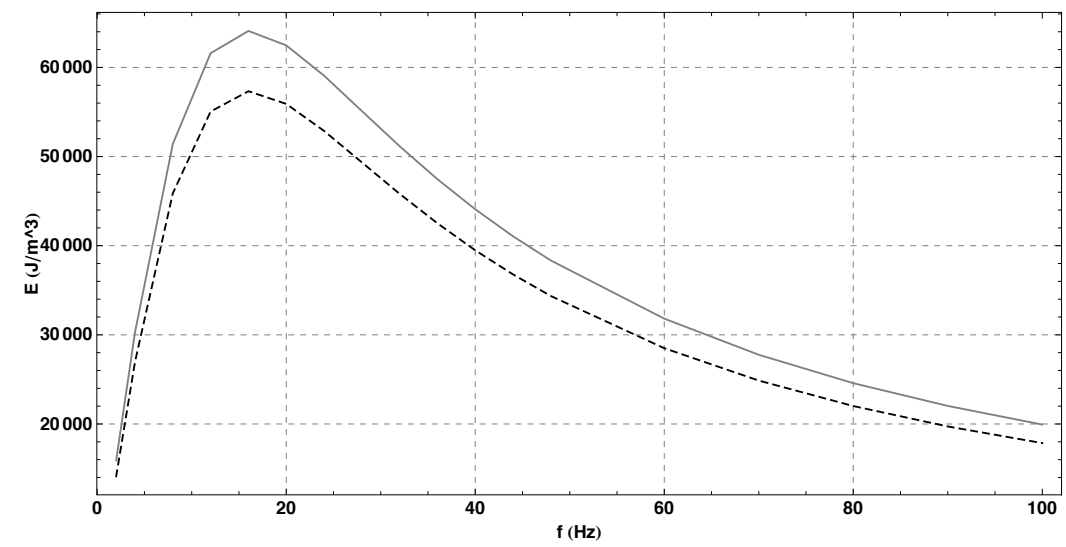

Figure 5: Energy dissipation per unit volume over a single period in the case of the Abaqus model (gray, continuous curve) and of the QLVE model (black, dashed curve) for simple shear. 


\section{Acknowledgements}

We thank the Università di Roma "La Sapienza" (Italy), the CNRS (France), and the Royal Society (UK) for their support.

\section{References}

Drapaca, C.S., Sivaloganathan, S., Tenti, G.: Nonlinear constitutive laws in viscoelasticity, Math. Mech. Solids 12, 475-501(2007)

Hibbit, D., Karlsson, B., Sorensen, P.: ABAQUS/Theory Manual version 6.7, Hibbitt, Karlsson \& Sorensen, Inc., Rhode Island (2007)

Johnson, G.A., Livesay, G.A., Woo, S.L-Y., Rajagopal, K.R.: A single integral finite strain viscoelastic model of ligaments and tendons. ASME J. Biomech. Eng. 118, 221-226 (1996)

Pipkin, A.C., Rogers, T.G.: A non-linear integral representation for viscoelastic behavior, J. Mech. Phys. Solids 16, 59-74 (1968)

Rajagopal, K.R., Wineman, A.S.: A quasi-correspondence principle for QuasiLinear viscoelastic solids Mech. Time-Depend. Mater. 12, 1-14 (2008)

Wineman, A.S.: Large axially symmetric stretching of a non-linear Int. J. Solids Struct. 8, 775-790 (1972) 